\title{
EDITORIAL
}

\section{Continuous Positive Airway Pressure Compliance: Addressing the Elephant in the Room}

\author{
Rohit Kumar ${ }^{1}$, Siddharth R Yadav ${ }^{2}$ \\ Indian Journal of Sleep Medicine (2021): 10.5005/jp-journals-10069-0084
}

Diagnosing obstructive sleep apnea (OSA) and managing it effectively has been an important therapeutic goal for all sleep clinicians. Though OSA had been known for years, its effective management using positive pressure administered noninvasively was reported 40 years ago. In 1981, Sullivan et al. published an article in Lancet describing the use of continuous positive airway pressure (CPAP) to treat sleep apnea.'

Sleep medicine specialists have diagnosed multiple cases over the last few years. Research done in the last few decades has shown that OSA is not only a common condition but also associated with various other cardiometabolic and neurological comorbidities, and optimum management of any patient requires that all conditions (including OSA) must be effectively treated. Treatment with CPAP has become the first line of management of patients with OSA.

The positive impact of the CPAP therapy has been shown by the dramatic improvement in the patients' self-perceived sleep quality, increased levels of alertness during the day, and the reduction in the daytime sleepiness with consequent enhanced work performance, lesser accidents, and better mood/attitude. ${ }^{2}$ In addition, CPAP usage has also been associated with decreased blood pressure and improved ejection fraction.

However, it has been observed that not all patients prescribed CPAP show similar improvements. It has been speculated that this might be because the pathophysiological changes may be irreversible or the patient may be noncompliant with CPAP treatment.

CPAP compliance has been defined as using the therapy for an average of 4 hours a night for at least $70 \%$ of the nights. However, this definition of using CPAP only for 4 hours is an arbitrary one. If a patient is using CPAP for 100 days and does not use it for 30 days during this period, he or she is still classified as being compliant to treatment! Also, if a patient has rapid eye movement (REM)-predominant OSA, he or she is likely to have more apnea events in the latter half of the sleep when REM sleep predominates. If such a patient is using the CPAP only in the initial 4 hours, the likely therapeutic effects are going to be suboptimal. The treatment also assumes that the use of CPAP will eliminate all OSA events; though optimal titration is always not possible. Hence, the definition of compliance though frequently quoted is not a logically or scientifically valid definition.

Despite such a "lenient definition," up to half of the patients are reported to be noncomplaint to treatment. ${ }^{3}$ This noncompliance with CPAP treatment has restricted its effective use and may affect the positive outcomes expected in patients whose OSA is completely managed. ${ }^{4}$ In this regard, multiple measures have been suggested to optimize the use of CPAP. Primary among them is educating the patient about the nature of the disease, health issues related to OSAS, and how CPAP can reverse many of the
${ }^{1}$ Department of Pulmonary and Critical Care Medicine, VMMC and Safdarjung Hospital, New Delhi, India

${ }^{2}$ Department of Pulmonary Medicine, VPCl, New Delhi, India

Corresponding Author: Rohit Kumar, Department of Pulmonary and Critical Care Medicine, VMMC and Safdarjung Hospital, New Delhi, India, e-mail: dr.rohitkumar@gmail.com

How to cite this article: Kumar R, Yadav SR. Continuous Positive Airway Pressure Compliance: Addressing the Elephant in the Room. Indian J Sleep Med 2021;16(4):95-96.

Source of support: Nil

Conflict of interest: None

problems. This has to be done at the earliest and requires frequent reiterations during regular follow-up visits. It is important to involve not just the patient but also the bed-partner in the patient-education program. It is advisable to educate the patient about CPAP therapy, the machine unit, the tubings, and interface available and give an opportunity to try it before the actual sleep study/titration. In patients with claustrophobia or excessive anxiety regarding the CPAP device, an abbreviated cardiorespiratory study may be tried prior to the titration..$^{5}$ During this, patients work with a technologist with a minimal hookup to polysomnography equipment. This helps them get a first-hand experience of what the CPAP feels like and acclimatize to it.

In view of the various types of devices available, the physician and the patient should be involved in selecting the most appropriate device. The consensus from the American Academy of Sleep Medicine stated that auto-CPAP may be specifically considered in cases of pressure intolerance, REM apnea, or positional apnea or when the in-lab PAP titration was inadequate, when the patient is undergoing a planned weight loss (bariatric surgery). ${ }^{6}$ A Cochrane review reported that in adults with moderate to severe sleep apnea auto-CPAP probably increases machine usage by about 13 minutes per night; this is not a clinically relevant improvement in CPAP usage. ${ }^{7}$ However, the current reductions in the price of auto-CPAP in India have made it affordable, and hence, it is frequently prescribed.

Another innovation that has been tried is the pressure-relief PAP, in which there is a reduction in pressure at the very end of inspiration and beginning of expiration so patients do not have to breathe against the prescribed therapeutic pressure immediately. Before the end of expiration, the pressure is restored to its therapeutic level to keep the airway open at the beginning of the next inspiration. However, this technique has not been shown to improve the compliance. ${ }^{8}$

The sleep center should have various types and makes of the masks and headgear. Sleep technicians need to work with 
the patient to get the best choice/fit. Before applying the CPAP, issues related to mouth-breathing, leaks, swallowing, and talking should be discussed. Patients with claustrophobia may tolerate nasal pillows or nasal prongs more than the nasal mask. Pillows or prongs are also preferred by patients who have a moustache or are missing dental support. An oronasal mask may be a better choice for patients with nasal congestion or a persistent mouth leak. It is important to ensure that the head straps should not be too tight and should not cause discomfort.

The initiation of the CPAP should be done under the supervision of a physician or trained technician and patient allowed to wear the interface at the office. They need to be assured that despite the noise, they will be able to sleep and will soon adjust to sleeping with the device on. It would be advisable to call the patient for the initial follow-up after a few days and assess any emergent issues and concerns. Correcting a problem up front is essential for good CPAP compliance.

In patients who are unable to tolerate having the CPAP device, they may be instructed about mask desensitization. ${ }^{9}$ In this, the patient is initially instructed to breathe calmly with a CPAP machine with a mask held in front of the face (not tied) for 1-2 minutes. This may be repeated several times during the day. Once comfortable with this, they should then start wearing the mask (unattached to the CPAP) for 10-15 minutes while doing normal activity. This too has to be done several times until they are acclimatized to the sensation of having the mask on the face. Subsequently, they are advised to try using CPAP during short naps during the day and once comfortable with its use, they can start using it regularly at night.

In a patient who complains of nasal congestion or sinusitis, nasal sprays with corticosteroids and antihistamines may be used. In addition, patients may complain of drying of the mouth and nose. Though the evidence in favor of the use of heated humidification is lacking ${ }^{10}$ specialists frequently prescribe it, especially in areas where the climate is often dry, as patients report fewer complaints of mucosal dryness when using humidification.

Further follow-up is needed on a regular basis to assess the usage of the equipment (hours of use and hours of application), check the machine settings, and ensure the interface (mask, pillows, etc.) is in good condition. This provides an opportunity to reinforce the need for compliance with the prescribed treatment. In addition, the regular maintenance of equipment should be emphasized.

Patients who are compliant may be invited to join support groups to share their experience with new patient being initiated on treatment. This allows patients to share tips on how to cope with using CPAP, anecdotal discussions regarding the effect of CPAP on health, reinforcement of the education offered on CPAP, and bearing each other's burdens.

In addition, the effective use of motivational strategies can help in promoting adherence to CPAP in patients with OSAS. Behavioral change is a complex process involving three specific constructs: (a) readiness to change, (b) perceived importance of change, and (c) confidence in one's ability to change. Effective targeting of these elements may help in patients accepting CPAP treatment. ${ }^{11}$

In coronavirus disease (COVID) times, where regular follow-up may be hampered, the CPAP usage data may be accessed remotely and telemedicine ${ }^{12}$ can be used as an important adjunct to improving compliance and suggesting techniques to overcome barriers to its daily use.

CPAP therapy is the gold standard for treatment available for patients with moderate to severe OSA, though poor adherence to PAP therapy is a persistent problem. The simple measures suggested above can affect the compliance with CPAP and may improve the patient's quality of life. It is important that during all visits, a frank discussion should be encouraged, and it is equally important that the sleep physician maintains a positive approach (e.g., explain that though many have problems in initially accepting the CPAP therapy, but they should not refuse it and it benefits them if in the long run).

\section{References}

1. Sullivan CE, Issa FG, Berthon-Jones M, et al. Reversal of obstructive sleep apnoea by continuous positive airway pressure applied through the nares. Lancet 1981;1(8225):862-865. DOI: 10.1016/ s0140-6736(81)92140-1.

2. Giles TL, Lasserson TJ, Smith BJ, et al. Continuous positive airways pressure for obstructive sleep apnoea in adults. Cochrane Database Syst Rev 2006;(1):CD001106. DOI: 10.1002/14651858.CD001106.pub2.

3. Kribbs NB, Pack Al, Kline LR, et al. Objective measurement of patterns of nasal CPAP use by patients with obstructive sleep apnea. Am Rev Respir Dis 1993;147(4):887-895. DOI: 10.1164/ajrccm/147.4.887.

4. Rotenberg BW, Murariu D, Pang KP. Trends in CPAP adherence over twenty years of data collection: a flattened curve. J Otolaryngol Head Neck Surg 2016;45(1):43. DOI: 10.1186/s40463-016-0156-0.

5. Krakow B, Ulibarri V, Melendrez D, et al. A daytime, abbreviated cardio-respiratory sleep study (CPT 95807-52) to acclimate insomnia patients with sleep disordered breathing to positive airway pressure (PAP-NAP). J Clin Sleep Med 2008;4(3):212-222. PMID: 18595433; PMCID: PMC2546453.

6. Morgenthaler TI, Aurora RN, Brown T, et al. Practice parameters for the use of autotitrating continuous positive airway pressure devices for titrating pressures and treating adult patients with obstructive sleep apnea syndrome: an update for 2007. An American Academy of Sleep Medicine report. Sleep 2008;31(1):141-147. DOI: 10.1093/ sleep/31.1.141.

7. Kennedy B, Lasserson TJ, Wozniak DR, et al. Pressure modification or humidification for improving usage of continuous positive airway pressure machines in adults with obstructive sleep apnoea. Cochrane Database Syst Rev 2019;12(12):CD003531. DOI: 10.1002/14651858. CD003531.pub4.

8. Sunderram J, Ayappa I, Lu SE, et al. PAP adherence and nasal resistance. A randomized controlled trial of CPAPflex versus CPAP in World Trade Center responders. Ann Am Thorac Soc 2021;18(4): 668-677. DOI: 10.1513/AnnalsATS.202009-11610C.

9. Means MK, Edinger JD. Graded exposure therapy for addressing claustrophobic reactions to continuous positive airway pressure: a case series report. Behav Sleep Med 2007;5(2):105-116. DOI: 10.1080/15402000701190572.

10. Haniffa M, Lasserson TJ, Smith I. Interventions to improve compliance with continuous positive airway pressure for obstructive sleep apnoea. Cochrane Database Syst Rev. 2004;(4):CD003531. DOI: 10.1002/14651858.CD003531.pub2.

11. Rapelli G, Pietrabissa G, Manzoni GM, et al. Improving CPAP adherence in adults with obstructive sleep apnea syndrome: a scoping review of motivational interventions. Front Psychol 2021;12(3266):705364. DOI: 10.3389/fpsyg.2021.705364.

12. Sparrow D, Aloia M, Demolles DA, et al. A telemedicine intervention to improve adherence to continuous positive airway pressure: a randomised controlled trial. Thorax 2010;65(12):1061-1066. DOI: $10.1136 /$ thx.2009.133215. 